\title{
An Electro-Optic Data Communication System for the Delft Parallel Processor.
}

\author{
E.E.E. Frietman, A.B. Ruighaver *
}

\begin{abstract}
The Delft Parallel Processor (DPP), which has already been operational since 1981, is part of a long term research project for the application of a large-scale Multiple Instruction stream, Multiple Data stream (MIMD) architecture in the field of scientific computing. As a result of this project a second modern version, the DPP84, equipped with up to 16 Processing Elements (PE's), is now operational and has been installed at several sites inside and outside the Technical University Delft. The most important part of the DPP is its communication system, which is based on a "multiple broadcast" concept. This allows all the PE's to be fully interconnected through fast parallel data channels. To realize a large-scale next-generation MIMD supercomputer in which all PE's are fully interconnected, a different technical approach to this communication system is needed. This paper introduces the concept of an Electro-Optic Communication System (EOCS).In order to be able to implement a high-bandwidth parallel interconnection structure, the application of flexible polymer fiber-optic cables is considered.
\end{abstract}

\section{INTRODUCTION}

Since 1981, research at the Technical University Delft on topics such as parallel architecture and algorithms has been supported by an operational MIMD structured parallel processor, the Delft Parallel Processor [1], [2]. [3]. In a second version of this processor, the DPP84 [5], [9], which is realized in a configuration of up to 16 tightly interconnected Processing Elements (PE's), each PE can achieve a performance in the MFLOP range.

Owing to a grant from the Dutch Ministry of Education and Sciences a feasibility study is now in progress to investigate the realization of a next-generation MIMD supercomputer, in which each PE performs at an effective double-precision speed of more than 10 Million FLOPS (MFLOPS).
The biggest problem in both the design and the application of large-scale parallel computers is the way communication is accomplished between all the different processors. The Delft Parallel Processor is characterized as a multicomputer architecture; i.e. the total memory space of each PE only consists of local memory, while communication between PE's is handled by special message passing hardware. In contrast to a multiprocessor architecture, based on communication through shared variables in a common memory, most multicomputer architectures rely on a restricted topology in order to keep the cost of the applied communication hardware low. Although the number of processors in a multiprocessor architecture is limited, the absence of restrictions for getting access to an arbritrary shared memory partition allows the use of simple allocation strategies. As a result most users regard a multiprocessor as easy to program.

Communication in the DPP is based on a "multiple broadcast" concept. In this concept, memory is not shared in common, so that memory latency, caused by the arbitration necessary to maintain data integrity and consistancy, is absent. All PE's ( see Fig.1), each equipped with its own local memory, produce their results through their Output Buffers (OB's). The output data from all the PE's is transported by means of parallel data channels to the Input Buffers (IB's) in each PE, including its own. Hence, this particular design in which all PE's are fully interconnected by a multiple of parallel data channels, proves to be of tremendous importance for the programmability of the DPP [4]. [8]. [11].

The normal organization of a data transfer in this DPP communication system is characterized by a full synchronization of all data channels at the start of each transmission: the newspaper concept. A newspaper consists of all the values which are stored in the OB's of each PE. After transmission, this particular newspaper is available at the IB's of all the

(") Dr.A.B.Ruighaver is now with the University of Melbourne, Dept. of Computer Science, Parkville, Victoria 3052, Australia. 
PE's for retrieval of specific data subjects until the next issue is received. Hence, each PE always executes its own independant stream of instructions operating on local data either in the IB, or in the local memory in order to be able to produce new data for broadcasting to other PE's.

One important additional aspect of the use of broadcasting in a multicomputer architecture is the possibility to monitor the transfer of data without retarding the data processing or changing the computational structure of a parallel program.In the configuration of the DPP84 (see Fig.1) two special purpose PE's are implemented for automatic visualization of the data, which is transferred via the communication system. Because neither PE produces data to be used by other PE's, their outputs are not connected to the parallel data channels.

The on-line State Observation PE (SO/PE) performs postprocessing on selected variables and sends this data to a microprocessor controlled multi-channel real time window display. In this way several variables can be monitored during the execution of a parallel program.

The second particular PE, the Trajectory Observation PE (TO/PE), has the function of simultaneuously storing the trajectories of the variables produced, derived from the OB's and/or from the external inputs. The purpose of this is to allow observation to take place at a later point in time.

The architecture of the PE84 is extensively described elsewhere [2], [3], [7]. For now, it sulfices to emphasize the high performance of a PE in current and future Delft Parallel Processors. This performance is based on an analogy of its architecture to the Harvard architecture, which is often used in digital signal processing chips. The PE-program is stored in the Program Memory (PM), while data is stored in a separate memory, called the Variable Memory (VM). Furthermore, in contrast to the design of the PE in the DPP81, which has little internal parallelism, the PE-84 version possesses several concurrently operating functional units. The DPP84 is therefore considered to be a first step to a Distributed MIMD processor (DMIMD) [5], [6], [10].

Finally, the processing power and speed of the DPP84 has been improved by the implementation of a new arithmetic processor, the Inner Product Processor (IPP). The principle of the IPP is based on a hardware kernel capable of performing the multi-operand floating point operation : " $a+b$ " $c$ " [14]. The current design uses a commercially available WEITEK chipset, consisting of a 64 bit floating point multiplier (WTL1264) and ALU (WTL1265) combination.
In the next-generation Delft Parallel Processor the performance of a single low-cost PE is expected to exceed the 10 MFLOP range. At the same time the size of this multicomputer can expand to a hundred PE's or even more.

Our current aim is to develop a powerful parallel communication mechanism, which will be able to serve up to a thousand PE's, each of them performing at an increasing processing speed. This mechanism is expected to produce a large system-wide bandwidth, but not to dominate the cost of the system. Fortunately, the capabilities of optical communication technologies seem to be optimally "adjusted" to the communication architecture implemented in the Delft Parallel Processor.

\section{THE USE OF OPTICS IN NEXT_GENERATION SUPERCOMPUTERS}

The state of the art solution for the implementation of fast shared data and/or address busses is based on the multilayer back plane, consisting of paraliel conductors etched on a printed circuit board (PCB). This technology is only reliable; however, when used within short distances and when applied to a limited number of connections. The current generation of supercomputers therefore relies on carefully terminated coaxial interconnections for the transmission of data. Because this is only economical for a limited number of interconnections, the designer is often forced to arrange the data transport in a time-multiplexed way, thereby increasing the complexity of both the input and the output circuitry.

Replacing the coaxial cable by a fiber-optic connection results in a further increase in capacity.This is however limited by the technology available for the high speed digital senders and receivers necessary to perform last communications. Until quite recently the only practical choice in the field of high speed digital circuitry was the application of Emitter Coupled Logic (ECL) bipolar IC's, but their speed is far from sufficient.

The future technological edge surely belongs to the ultrahigh-speed GaAs digital IC's. The main advantage of the GaAs technology is that the internal energy band structure allows electrons to move through the substrate crystal lattice at a speed almost five times faster than in silicon and at lower voltages. The power dissipation is 5 to 10 times less than with ECL. With shift registers, counters and multipliers working at a speed of $1 \mathrm{~Gb} / \mathrm{s}$, digital GaAS IC's will not only form the basis for a new generation of optical links, but also for a new generation of ultra fast computers. Hence, the use of (time-multiplexed) optical point to point connections does not seem to be a solution in the 
realization of a next-generation supercomputer.

The use of optics will prove in general to be advantageous, especially when distances greater than $1 \mathrm{~mm}$ [13] have to be bridged. Other applications will take advantage of specific properties, where a signal has to be provided to a large number of electronic circuits with very little mutual difference in clock skew. In the case of a synchronized operation the use of optics for clock distribution to high speed digital components is strongly recommended [12].

The requirements for ultimate speed also inevitably lead to the design of optical computers. While High Electron Mobility Transistor (HEMT) technology, a relatively now entry in GaAs IC development, provides switching speeds of around 15 picoseconds, optical switches will practically be able to function at speeds of 1 picosecond or even less. Although some research projects have already produced bistable optical devices, large-scale application of this technology in a pure optical computer should not be expected in the near future.

The Electro-Optic Communication System described in the next chapter does not depend on future optical switching technologies. The communication architecture of the Delft Parallel Processor is based on local selection of the required data items out of an enormous amount of data, received from other PE's. Hence, next-generation DPP's can make use of the most important advantage of optics, its ability to create dense parallel interconnection networks, while simple electro-optic and opto-electric transducer circuits can be applied for sending and receiving the data.

\section{THE ELECTROOPTIC COMMUNICATION SYSTEM}

For the transmission of optical energy between a transmitter and several receivers both free space optics and optical wave guides may be used. Which medium should be used depends mostly on the distance to be bridged and on the number of parallel channels that are needed. In either case implementation of the interconnection network takes advantage of the fact that photons, unlike electrons, do not interact when they travel over a distance.

In free space optics, lenses, prisms and mirrors all have the property that they are able to transfer billions of space-resolvable points in parallel. In contrast to electrical busses the optical information can cross on the same plane without interacting, creating shorter data paths and more compact layouts.

There is, though a practical limit to the amount of parallel channels that can be created using optical

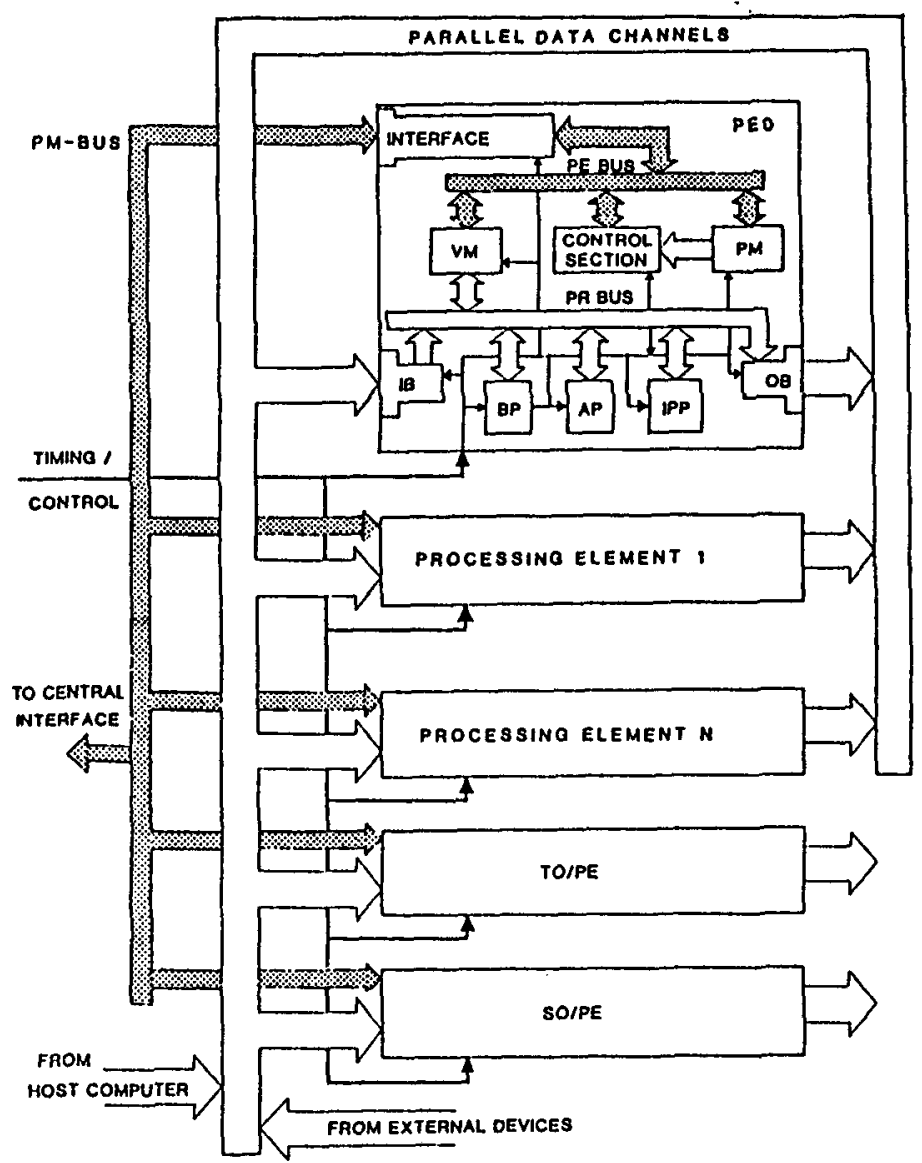

Fig.1 The multicomputer architecture of the DPP84.

waveguides. The greater the number of channels, the harder to align each single transmission channel with its transmitter and receiver in a distinct way. Hence, the most promising solution for the near future is the implementation of optical fibers in a multiple-broadcast communication system.

The proposed communication system is illustrated in Fig.2. The electrical signals from each $O B$ are converted by Light Emitting Diodes (LED's) into optical signals. The energy of each LED has to be distributed over a bundle consisting of 1000 multi-mode fibers. This bundle is transported through the back plane and finally split up into 1000 separate fibers, which in turn are connected to the corresponding bit locations in the IB's of each of the PE's.

Sand, the basic raw material for glass fibers, is not a scarce resource and the price of fiber waveguides is therefore not dependent on the costs of its material.While in recent years the telecommunications industry has shown a growing interest in the use of optical fibers for the transport of information, it is expected that due to the drop in prices and the 
improvement in production techniques, an increase in consumption will take place in the application of fibers in supercomputers.

It is realized, however, that the implementation of a. full parallel multiple-broadcast interconnection between a thousand PE's requires the routing of $64 * 1000 * 1000$ fibers. Fortunately, a fiber optic transmission system can be much smaller in size and lighter in weight than an electrical equivalent of the same bandwidth. Even with that amount of fibers the dimension of the optical fiber bulk is still manageable. The availability of Computer Aided Design / Computer Aided Manufacturing (CAD/CAM) techniques should therefore stimulate the realization of this rather ambitious goal of mutually connecting a thousand PE's, especially in the case of the use of optical fibers in this EOCS.
Another reason that makes the application of an optical transmission system so very attractive is that it is possible to choose a design which allows these special opto-electronic circuits to be replaced easily. Whenever technology improves one may also expect an increase in the processing speed in each PE. The investment in the improvement of a complete fiber back plane can then be kept low.

Still, the problem of a simultaneous conversion and parallel storage of the optical data has to be solved. To realize a high-bandwidth digtital signal link a special hardware component at the receiver side has to be developed. The design of such a component relies mainly on the requirements of a parallel loading and storage of 64,000 bits of information, in the case of

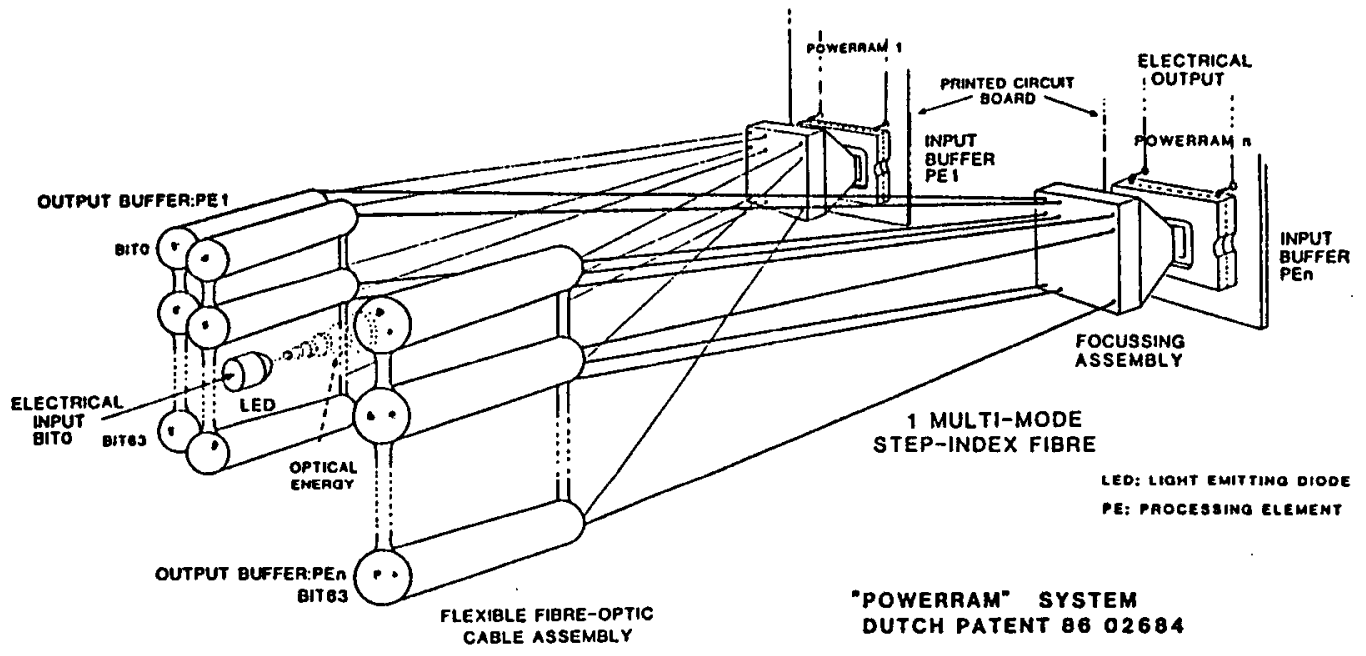

Fig.2 The Electro-Optic Communication System.

Even more important than the implementation of optics is the design of the extra components, such as transmitters for converting the electrical signals into light and receivers for performing the reverse conversion.

Not only will the cost of these components contribute significantly to the total cost of the $P E$, but the use of the current technology will also potentially be the bottleneck in the EOCS. Because the distances to be bridged in a future parallel processor will be in the order of 5 to 10 meters, both a small signal attenuation as well as a small signal dispersion have to be considered. Therefore, the speed of the EOCS will strongly depend on the speed of the input and output buffers. the implementation of 1000 PE's and the use of a computer word with a length of 64 bit. A proposal has been formulated for the development of a bipolar Parallel Optical Write, sequential Electrical Read Random Acces Memory (POWERRAM,TM; European patent 86.02684) based on a 1000 * 64 array of flip-flop elements. See Fig.3. The function of this POWERRAM is to convert the parallel presented information, which is conducted through the 1000 "64 multi-mode fibers into a collection of electrical signals with a resolution of 64 bits [15].

\section{DESIGN ASPECTS OF THE PROTOTYPE EOCS}

An attempt will be made to specify the requirements for a medium speed EOCS prototype 
implemented with optical fibers. Technical aspects with much more detail can be found elsewhere [15].

The transmitter side is realized using a specially designed array of high power LED's. At present, LED's show several drawbacks, like lower optical power coupling into a liber, relatively small modulation bandwidth and significant harmonic distortion. There are, however, many advantages like simpler fabrication, lower costs, higher reliability, less temperature dependence, simpler driving circuitry and the availability of a linear oulput versus current characteristic. The approximate rise time of the chosen LED will be in the order of 5 ns. It is estimated that about $10 \mathrm{uW}$ of power is coupled into the fiber.

The fiber to be used in the back plane is a polymer step index fiber with a core of 360 ucrons. The application of polymer instead of glass fibers includes the disadvantage of higher losses, which is, however, less important in an EOCS with short links of maximally 10 meters. Polymer fibers are simple to handle and less costly, properties of extreme importance in a large-scale EOCS.

For transporting the data through the optical fiber a Non-Return to Zero (NRZ) format is chosen, where the pulse duration is the reciprocal of the data rate. Theoretical calculations and simulations indicate that a single link could support a maximum bit rate of $\mathbf{5 6}$ $\mathrm{Mb} / \mathrm{s}$, which includes a rise time of $10 \mathrm{~ns}$ for the combination of the optical detector (PIN photodiode) and electronic circuitry in the POWERRAM. Since an NRZ format is used, the equivalent of the $3 \mathrm{~dB}$ optical bandwidth will be $28 \mathrm{MHz}$.

The quality of the complete transmission system, including the electro-optic and opto-electric transducers, is expressed in the probability of the occurrence of errors. Fluctuations in the pulse duration and the existence of noise usually determines the presence of errors. The amount of optical power reaching the optical detector therefore influences the Bit Error Rate (BER) of the complete system. When ideal conditions are assumed for the detector as well as for the transmitted binary signals, an approach to the overall power budget of the complete EOCS is estimated to be one bad bit out of 1 billion bits.

\section{RESULTS}

Measurements were performed on complete digital links, consisting of a cheap LED, 5 meters of polymer fiber, permanently glued to the LED on one side and to the POWERRAM on the other side. The connection was made by first precisely positioning and then carefully fastening the fibers to the chip. A perspex coupler was developed in order to make a permanent and more efficient coupling possible. Though designed for
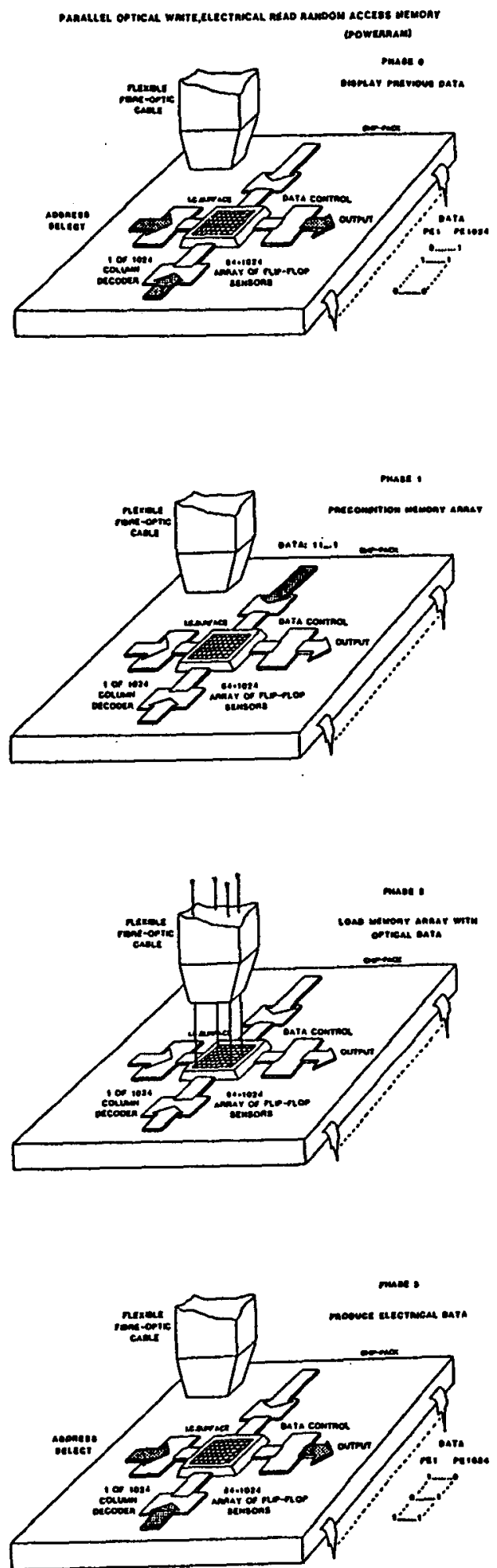

Fig.3 The four phases of initialization of a POWERRAM. 
operation at a speed of $10 \mathrm{Mb} / \mathrm{s}$, very accurate timing even demonstrates a data bit rate of $20 \mathrm{Mb} / \mathrm{s}$. Both figures state the maximum allowable repetition rate, namely the alternate transmission of a digital "1" and a " 0 ". The time elapsedbetween the triggering of the LED and the presence of valid data at the output of the POWERRAM is approximately $12 \mathrm{~ns}$. When these results are extrapolated to the future POWERRAM, equipped with an array of $64^{\circ} 1000$ storage elements, the data transfer will be in the $\mathrm{Tb} / \mathrm{s}$ range. By comparison : this equals the transmission of a text of about half $a$ million paperbacks per second, while sending four pages at the same time every 70 ns.

\section{FUTURE TRENDS}

The manufacture of LED's and PIN-photodiodes composed of compound GaAs (III-IV) combinations is well known. Integrating these opto-electronic devices with high density Si circuitry by depositing GaAs on top of a processed Si wafer and then building devices in it is the next step to the realization of components which will bridge the gap between optics and electronics. The addition of much more intelligence to the design of the POWERRAM, in order to increase the amount of parallelism in the next-generation DMIMD supercomputer ( the DPP8X), has already led to the development of a new opto-electronic device, a Parallel Optical Write, parallel Electrical Read Content Adressable Memory; the POWERCAM (TM; patent pending).

The DPP8X, which shows this high degree of interconnectability, could support the theoretical aspects in the modern concepts of computing theory: the neural network. Pioneered by John Hopfield of the California Institute of Technology, the idea behind the neural network concept shows a massive interconnection among neurons. In this network each individual computational unit, the neuron, directly communicates with thousands of others. This dense interconnectivity, which is evident in neural networks, is only feasible with optics [16].

Dr. Hyatt M.Gibbs, director of the optical circuitry cooperative at the University of Arizona has even stated that instead of performing arithmetic calculations, processing is done more or less in an associative parallel-reasoning way, which looks more like educated guesses in an on-line feedback situation. Joining the properties of associative memories, which recall an entire collection of data, while it is only stimulated by a partial set of information, the DPP $8 X$ equipped with a POWERCAM, implemented as an input buffer in the PEBX, offers the opportunity to do research on this specific topic [16].

\section{CONCLUSION}

In the case of a multicomputer architecture such as the Delft Parallel Processor, in which the electrical interconnections between the mutually connected PE's are replaced by optical links, the influence of the communication bottleneck on the processing speed can be diminished. The structure of the DPP, which supports a communication structure based on a multiple broadcast concept, is ideal for implementing optics. The application of an optical back plane (being a subpart of an Electro-Optic Communication System) in a next-generation DMIMD supercomputer (the DPPBX) promises a new breakthrough in applying more parallelism,while still having a reasonably low cost/performance ratio. Research in the near future will be dedicated to implementing fast parallel interconnections, through special opto-electronic circuitry (POWERCAM \& EOCS), and to performing parallel associative processing through dedicated hardware.

\section{ACKNOWLDGEMENT}

I am indebted to mrs. Susan Massotty for reading the paper and providing many helpful comments and clarification of the language. I also thank A.B. Ruighaver for his support and additional suggestions.

\author{
REFERENCES \\ [1] H.J.Sips, "Design aspects of a distributed MIMD \\ processor ", Ph.D.Thesis, Delft, Technical \\ University Delft, March 1984.
}

[2] H.J.Sips, "Philosophy behind the design and construction of the Delft Parallel Processor as a simulation tool ". Proc. SIMS 1982 Annual meeting, Trondheim, Norway, 1982.

[3] L.Dekker, "Parallel Processors in Engineering Sciences", Proc. Simulation in Engineering Sciences, IMACS, Nantes, 1983.

[4] S.W.Brok, L.Dekker, E.J.H. Kerckhoffs, A.B.Ruighaver, H.J.Sips," Architecture and programming of the MIMD structured Delft Parallel Processor ", Proc. First European Simulation Congress, Aachen, 1983. 
[5] J.H.M.Andriessen,S.W.Brok,L.Dekker,A.B.Ruighaver, H.J.Sips, "The Delft Parallel Processor, a distributed MIMD-processor ", Proc. SCS-summer Computer Conf., Boston, 1984.

[6] L.Dekker, " The Delft Parallel Processor toward a DMIMD-processor ", Workshop Compute Architecture, U.S.Naval Research/ London, London, 1984.

[7] L.Dekker, "Methodology based Parallel Digital Processors ", 2nd International Symp. on System Analysis and Simulation, DDR, Academy of Sciences, August 1985.

[8] A.B.Ruighaver," Hierarchical Programming and the DPP84 ", Ph.D.Thesis, Delft, Technical University Delft, December 1986.

[9] A.B.Ruighaver, " Design Aspects of the Delft Parallel Processor DPP84 and its programming system ". Computer Architecture News , vol.14, no.1, January 1987.

[10] L.Dekker, "Expandibility of an MIMD Multiprocessor system to a large size ". Proc. of the 1985 Simulation Conf., Norfolk, March 1985.

[11] S.W.Brok, E.J.H.Kerckholfs, " The Delft Parallel Processor in a simulation environment ",Proc. of the 1984 UKSC Conf. On Computer Simulation, London, U.K.1984.

[12] B.D.Clymer, J.W.Goodman, "Optical clock distribution to silicon chips ", Optical Engineering, Vol.26, no 10, October 1986.

[13] P.R.Haugen, S.Rychnovsky, A.Husain, "Optical interconnecls for high speed computing "Optical Engineering Vol.25, no.10, October 1986.

[14] E.E.E.Frietman, F.Bruggeman, " The Inner Product Processor ", 13th Symp. on Microprocessing and Microprogramming, EUROMICRO 87, Southsea Portsmouth, United Kingdom, September 1987.(accepted for publication)

[15] E.E.E.Frietman, W. van Nifterick, " The application of OPTRONICS in the field of High Speed Parallel Processing '. LASER \& OPTRONICS, Las Vegas, Nevada, U.S.A. , 1987. (accepted for publication).

[16] J.Hecht, "Optical Computers may mimic Brain ", LASER - OPTRONICS, Las Vegas, Nevada, U.S.A., July 1987. 